\title{
Absolute Collision Cross Sections for Low Energy Electron Scattering from NO: The Role of Resonances in Elastic Scattering and Vibrational Excitation
}

\author{
M. Jelisavcic, R. Panajotovic, and S. J. Buckman \\ Atomic and Molecular Physics Laboratories, Research School of Physical Sciences and Engineering, \\ Australian National University, Canberra, ACT, Australia 0200 \\ (Received 17 February 2003; published 20 May 2003)
}

\begin{abstract}
Absolute measurements of elastic scattering and vibrational excitation of the NO molecule by low energy electron impact $(0.4-2.5 \mathrm{eV})$ are presented. They show that previous estimates of these cross sections may be in error by as much as a factor of 3 and provide compelling evidence for a reassessment of the balance between elastic scattering and vibrational excitation at incident energies below $2 \mathrm{eV}$. They also confirm the critical contribution that intermediate negative ion resonances $\left(\mathrm{NO}^{-}\right)$make to the various scattering processes for this molecule at low incident energies.
\end{abstract}

DOI: 10.1103/PhysRevLett.90.203201

PACS numbers: $34.80 . \mathrm{Gs}, 34.80 . \mathrm{Bm}$

Nitric oxide (NO) is a remarkable diatomic molecule that plays an important role in a wide range of chemical and physical processes. These include its well-known role in the atmosphere, e.g., dayglow and auroral processes, its contribution to photochemical smog, and an increasing realization of the critical role it plays in many human biological processes. Many of these processes, but, in particular, those of atmospheric relevance, are strongly governed by the way in which NO interacts with radiation. In this regard, its structure, and that of the bound and quasibound states of its negative ion $\mathrm{NO}^{-}$, are of particular importance in determining the role that low energy electron scattering plays in these processes.

NO is an open shell molecule with a small dipole moment $(0.15 \mathrm{D})$. It has a stable negative ion $\left(\mathrm{NO}^{-}\right.$ ${ }^{3} \Sigma^{-}$), with an electron affinity of only $24 \mathrm{meV}$ [1], which supports low-lying ${ }^{1} \Delta$ and ${ }^{1} \Sigma$ excited states and which, by analogy with its isoelectronic neighbor $\mathrm{O}_{2}$, lie within a few $\mathrm{eV}$ of the ground electronic state. For all but the (bound) ground vibrational level of the ground electronic state of $\mathrm{NO}^{-}$, the excited states of the negative ion manifest themselves as resonances in electron scattering cross sections, and it has been well documented that these states have a major effect on the scattering process. Early investigations [2,3] demonstrated a strong series of resonances which influenced, even dominated, both the elastic scattering and vibrational excitation cross sections. Other studies (eg., [4]) showed that these features, extending well above $1 \mathrm{eV}$ in energy, were in fact due to at least two quasivibrational series of resonances belonging to the ${ }^{3} \Sigma^{-}$ground state and ${ }^{1} \Delta$ excited state of the negative ion. Subsequent analysis [5] and a number of very high resolution experiments [6,7] revealed accurate figures for the electron affinity, the total scattering cross section, and the molecular constants for each of the excited states. One of these [7] also revealed the extraordinary extent to which scattering via these resonant states effects even the grand total scattering cross section.
It is surprising that throughout all of this detailed and important work on the nature of the resonances, there has been no concerted effort to establish the absolute values of the elastic and various vibrationally inelastic scattering cross sections for the NO molecule and, in particular, the quantitative role that the resonances play. Indeed, at energies below $5 \mathrm{eV}$, there are no accurate, direct measurements of the absolute cross sections for vibrational excitation.

In a recent publication, Josic et al. [8] have performed a comprehensive analysis of the body of previous experimental work, together with their own electron transport data for the transverse diffusion coefficient in NO, to derive a self-consistent set of electron scattering cross sections (grand total, integral elastic, integral vibrational, dissociation, electronic excitation, ionization) for the NO molecule. They found that their vibrational (0-1) excitation cross section, for example, was about a factor of 40 greater than the only previous estimate, that of Spence and Schulz [2]. It is not surprising that among the conclusions to this work was the observation that the low energy cross sections, particularly those for vibrational excitation, were not at all well understood and that further experimentation was warranted.

In this Letter we present what we believe are a comprehensive set of absolute cross sections for elastic scattering and the first few vibrational excitation modes $(0-1$, $0-2$ ) of NO at energies between 0.4 and $2.5 \mathrm{eV}$. They provide some interesting comparisons and contrasts to the previous work, including that of Josic et al..

The experimental data described here were measured on a crossed electron-molecular beam spectrometer. It consists of an electron monochromator employing electrostatic electron optical elements and a hemispherical energy selector, and a similar electron analyzer which is used to collect scattered electrons as a function of angle and energy. The apparatus has been described in significant detail in previous publications (eg., [9]). The energy 
resolution of the incident electron beam was $\sim 40 \mathrm{meV}$ and that of the overall spectrometer about $55 \mathrm{meV}$. The absolute value of the incident energy, of great significance to the resonance measurements described here, was determined by simultaneous calibration against the wellknown positions of the quasivibrational $\mathrm{N}_{2}^{-}$resonance structure in elastic scattering from $\mathrm{N}_{2}$ at around $2 \mathrm{eV} \mathrm{[10].}$

The present cross sections are measured using two modes of data collection. In one, the incident and scattered energies are fixed and the scattering angle is varied to enable measurements of angular differential cross sections (DCS) for both elastic scattering and vibrational excitation. In the other mode, the scattering angle is fixed and the incident energy is varied while the analyzer is tuned to detect either elastic or inelastically scattered electrons, enabling differential excitation functions (EF) to be measured. For either mode of operation the absolute values are determined by using the relative flow technique (e.g., [11]) in which the scattering intensity for elastic or inelastic processes in NO are compared to the elastic scattering intensity for helium. These comparisons are done under identical scattering conditions and are made at each energy and angle for which the present results are given. The He cross sections of Nesbet [12] are used as the absolute calibration standard. To ensure the integrity of the absolute cross sections for vibrational excitation, the postcollision zoom lenses on the electron analyzer are independently optimized for the transmission of both elastic and inelastically scattered electrons, such that the ratio of scattered signal between elastic and inelastic processes reflects the ratio of the cross sections.

Examples of EF's for elastic scattering and vibrational excitation $(v=0-1)$ are given in Figs. 1 and 2, respectively. Also shown in these figures are the cross sections obtained using the angular DCS mode of data collection

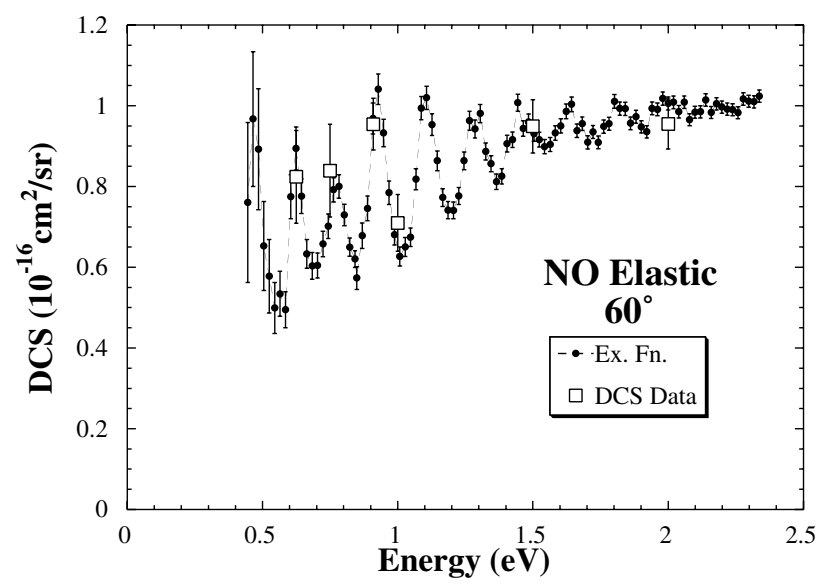

FIG. 1. Absolute elastic excitation function for $\mathrm{NO}$ at a scattering angle of $60^{\circ}$. The open squares are absolute values obtained at specific energies from the DCS mode. The error bars on these latter points give an indication of the absolute uncertainty. at several discrete energies. The error bars on these latter data points indicate the absolute uncertainty on the cross sections in both cases. There is clearly good agreement between the data obtained with the two, quite different, modes of operation of the spectrometer.

In Fig. 1 the resonances due to excitation of vibrational modes of the ${ }^{3} \Sigma^{-}$and ${ }^{1} \Delta$ negative ions states are clearly visible in the elastic scattering spectra and have a marked, even dominant, effect on the magnitude of the DCS at all of the angles we have investigated. The positions and widths of these resonances are consistent with earlier observations [3]. The two lowest lying features are vibrationally excited levels of the ground ${ }^{3} \Sigma^{-}$state of $\mathrm{NO}^{-}$and they are narrower than all of the higher energy features which are due to the ${ }^{1} \Delta$ and (perhaps) ${ }^{1} \Sigma$ states. The role of the latter state has not been clearly identified in previous work. While some of the higher lying features observed in our spectra have different profiles and widths than those in the vicinity of $1 \mathrm{eV}$, the possibility of interference between the various channels cannot be ruled out as the major cause of these variations. The new information which is contained in this and the other similar spectra is the measurement of the contribution that these resonances make to the absolute elastic scattering cross section.

Similarly, in Fig. 2 the results for the excitation of the $v=1$ vibrational mode of the NO molecule (threshold energy $=0.233 \mathrm{eV}$ ) are shown for a scattering angle of $90^{\circ}$. Here the resonant effects are even more marked with most of the vibrational excitation apparently occuring via the excitation of the negative ion. It is interesting to note the broader profiles at higher energies and the almost complete lack of resonant intensity at around $1.1 \mathrm{eV}$. The resonances in this channel also exhibit different profiles and positions to those in elastic scattering. Once again the results are consistent with the earlier, relative results [3] with regard to the details of the

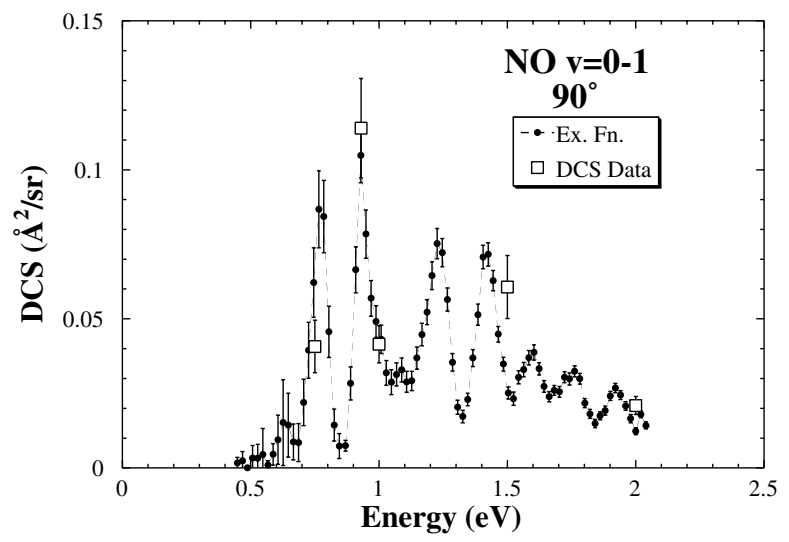

FIG. 2. Absolute vibrational $\left(v=0-1, E_{\mathrm{th}}=0.233 \mathrm{eV}\right)$ excitation functions for $\mathrm{NO}$ at a scattering angle of $90^{\circ}$. The open squares are absolute values obtained at specific energies from the DCS mode. 
resonance positions and relative magnitudes. Similar measurements have also been made at a range of angles for the $v=2$ vibrational mode (threshold energy $=$ $0.462 \mathrm{eV}$ ) but for reasons of brevity they are not shown here.

The principal object of the present work was to provide a set of integral elastic and vibrational excitation cross sections, based on absolute scattering measurements. To convert the set of differential excitation cross sections, examples of which are shown in Figs. 1 and 2, to integral cross sections (ICS) we have used the angular DCS measurements, which we do not show for reasons of brevity, at a number of discrete energies, between 0.625 and $3 \mathrm{eV}$, to assist in establishing the value of the ICS at these fixed energies. This has been done by using a multiparameter phase shift analysis technique [13] to fit the measured DCS, extrapolate it to forward and backward angles, and then obtain the absolute ICS by integration of the DCS. This gives us a number of discrete values $(\sim 1-5)$ of the ICS for each scattering process to assist in scaling the measured excitation functions. The major assumption that we have made in doing this is that the energy dependence of the ICS is similar to that of the differential excitation function measurements. As there are only small differences between the measured EF's at the various scattering angles, we believe that this is a reasonable assumption.

The set of scattering cross sections that result from this process is shown in Figs. 3-5, for energies between 0.4 and $3 \mathrm{eV}$. In Fig. 3 the integral elastic cross section is shown and compared to that of Josic et al. [8]. There are no significant differences between the two cross sections. [We note that the corresponding cross section which is shown in Fig. 2 of Josic et al. is in fact only an estimate of the resonant contribution to the elastic integral cross section, which is only about $50 \%$ of the total (resonant + direct) elastic cross section in the energy region around

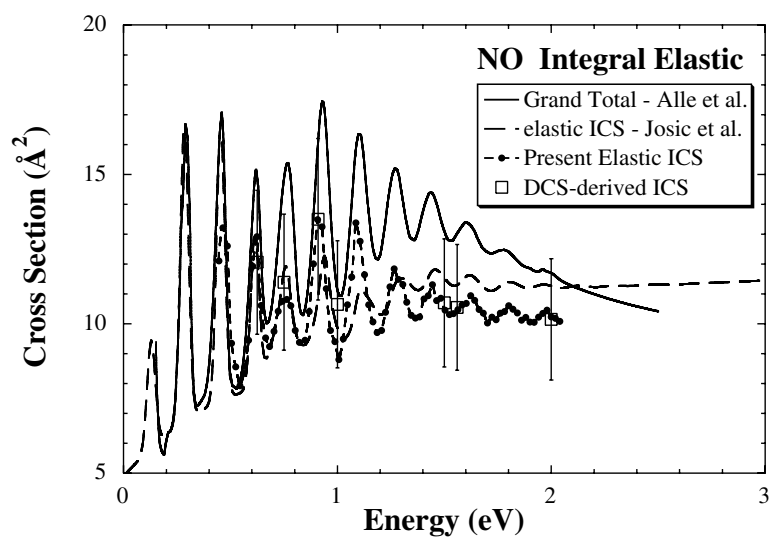

FIG. 3. Integral elastic cross section for NO. The present results are compared to the cross section of Josic et al. and the grand total cross section. Note that the zero of the plot has been displaced.
$1 \mathrm{eV}$.] Also shown in this figure is the measured grand total cross section of Alle et al. [7].

The ICS for vibrational excitation ( $v=1$ and 2$)$, derived from the excitation function measurements, are shown in Figs. 4 and 5, respectively. Also shown in these figures are the values of the ICS derived at discrete energies from the phase shift analysis of the data obtained using the DCS mode of operation. As discussed above, these values have been used, in part, in obtaining the absolute ICS for the excitation functions. It is for these vibrational cross sections that significant differences emerge between the present measurements and previous determinations. While we see (Fig. 4) a similar overall shape for the $v=0-1$ vibrational ICS, the present measurements are about a factor of 3 lower in magnitude than those of Josic et al. in the region of $1 \mathrm{eV}$. We also observe significantly less vibrational excitation, at energies below the large resonance peak at about $0.75 \mathrm{eV}$, than is evident in the cross section of Josic et al. Similarly, for the $v=$ 0-2 cross section (Fig. 5), the present measurements are both different in shape and significantly smaller in magnitude than the cross sections of Josic et al. In particular, they also indicate that there is no significant excitation of the $v=2$ level at energies below about $1 \mathrm{eV}$.

The present cross section measurements indicate that recent estimates of vibrational excitation in NO (0-1 and 0-2 modes) may be too large by as much as a factor of 3 . It is quite possible that some of these differences stem from the fact that the Boltzmann analysis [8] has been carried out with a set of electron transport data comprising accurate diffusion coefficient data, but with drift velocity data which only covers a limited range of $E / N($ electric field over gas number density) values and which is of uncertain accuracy. The additional constraint that would be provided by accurate and more extensive drift velocity data, were it available, may well

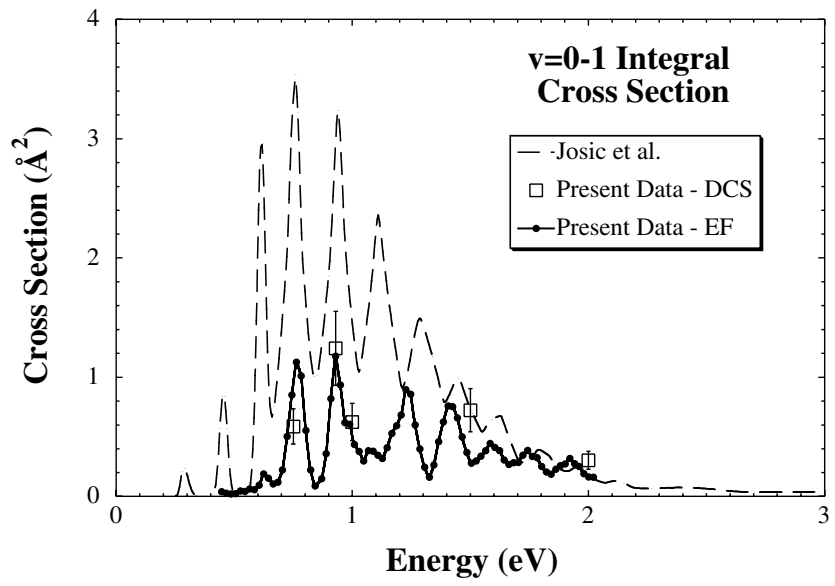

FIG. 4. Absolute integral cross section for the $v=0-1$ $\left(E_{\mathrm{th}}=0.233 \mathrm{eV}\right)$ vibrational excitation of NO. Present results from the two modes of data collection are shown and compared with the cross section of Josic et al. 


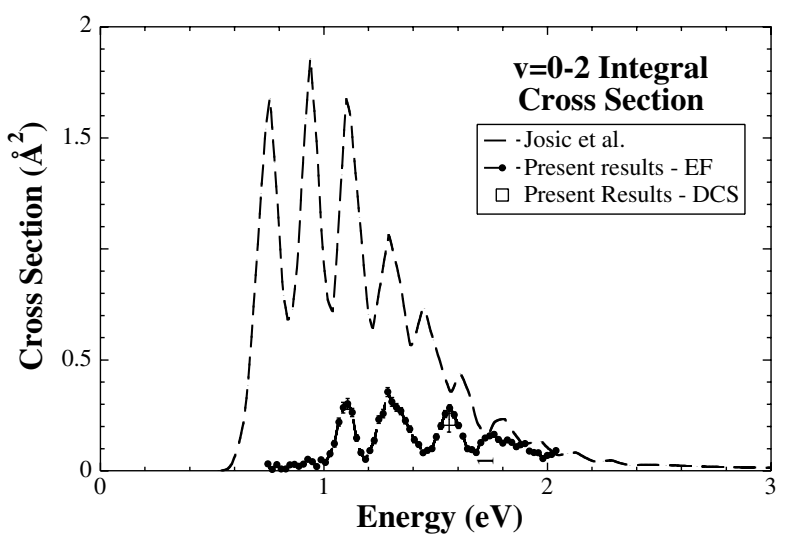

FIG. 5. Absolute integral cross section for the $v=0-2$ $\left(E_{\mathrm{th}}=0.462 \mathrm{eV}\right)$ vibrational excitation of NO. Present results from the two modes of data collection are shown and compared with the cross section of Josic et al.

be important. Nonetheless, such differences are significant and would clearly have a bearing on the modeling of processes where NO plays an important role, such as auroral emissions [14]. In particular, NO plays a pivotal role in the energy balance of our atmosphere through its ir emissions from fundamental and first and higher order overtones in its ground electronic state. The cross sections for vibrational excitation that we have measured clearly impact on an accurate description for this phenomenon.

This situation also provides a challenge for contemporary scattering theory. There are presently no theoretical estimates with which we can compare in this important energy region where resonant enhancement appears to play a vital role. Although a relatively small diatomic molecule, NO poses specific and difficult challenges for scattering theory with its open shell structure, nonzero dipole moment, and complex negative ion spectrum. We hope that the present measurements may stimulate some theoretical interest in these issues.

It is a pleasure to acknowledge Professor Zoran Petrovic of the Institute of Physics, Belgrade, and his colleagues, for alerting us to this issue and for his continued interest in the project.

[1] M.W. Siegel, R. J. Celotta, J. M. Hall, J. Levine, and R. A. Bennett, Phys. Rev. A 6, 607 (1972).

[2] D. Spence and G. J. Schulz, Phys. Rev. A 3, 1968 (1971).

[3] M. Tronc, A. Heutz, M. Landau, F. Pichou, and J. Reinhardt, J. Phys. B 8, 1160 (1975).

[4] P. D. Burrow, Chem. Phys. Lett. 26, 265 (1974).

[5] D. Teillet-Billy and F. Fiquet-Fayard, J. Phys. B 10, L111 (1977).

[6] J. Randell, S. L. Lunt, G. Mrotzek, D. Field, and J. P. Ziesel, Chem. Phys. Lett. 252, 253 (1996).

[7] D. T. Alle, M. J. Brennan, and S. J. Buckman, J. Phys. B 29, L277 (1996).

[8] L. Josic, T. Wroblewski, Z. Lj. Petrovic, J. MechlinskaDrewko, and G. P. Karwasz, Chem. Phys. Lett. 350, 318 (2001).

[9] J. C. Gibson, L. A. Morgan, R. J. Gulley, M. J. Brunger, C. T. Bundschu, and S. J. Buckman, J. Phys. B 29, 3197 (1996).

[10] K. Rohr, J. Phys. B 10, 2215 (1977).

[11] M. J. Brunger and S. J. Buckman, Phys. Rep. 357, 215 (2002).

[12] R. K. Nesbet, Phys. Rev. A 20, 58 (1979).

[13] R. Panajotovic, M. Kitajima, H. Tanaka, M. Jelisavcic, J. Lower, L. Campbell, M. J. Brunger, and S. J. Buckman, J. Phys. B 36, 1615 (2003).

[14] D. C. Cartwright, M. J. Brunger, L. Campbell, B. Mojarrabi, and P. J. O. Teubner, J. Geophys. Res. 105, 20857 (2000). 